\title{
Analysis of Phase-Inversion Waves in In-and-Anti-Phase Synchronization on Coupled Van der Pol Oscillators as a 2D Lattice by Using an Actual Circuit
}

\author{
Yoshihito Todani $^{\dagger}$, Seiko Kunihiro ${ }^{\dagger}$, Masayuki Yamauchi ${ }^{\dagger}$ and Yoshifumi Nishio \\ $†$ Department of Electronics and Computer Engineering, \\ Hiroshima Institute of Technology \\ 2-1-1,Miyake,Saeki-ku,Hiroshima,Japan \\ Email: todani4411@gmail.com \\ $\$$ Department of Electrical and Electronic Engineering, \\ Tokushima University \\ 2-1,Minami-Josanjima,Tokushima,Japan
}

\begin{abstract}
-
In this paper, we observe phase-inversion waves which simultaneously propagate to horizontal direction and vertical direction in in-and-anti-phase synchronization by using actual circuit experiments and numerical experiments. We observe transitions of propagation velocities by changing coupling parameters between two adjacent oscillators. We clarify a reflection mechanism of the phase-inversion waves at a corner when there are in-and-anti phase synchronization for vertical direction and in-phase synchronization for horizontal direction.
\end{abstract}

\section{Introduction}

Synchronization phenomena are studied by many researchers. The synchronization phenomena are observed at everywhere of the natural world, and the phenomena are used for human society. For example, the phenomena are used to communicating system, laser, and so on[1]-[2]. The synchronization phenomena can be observed in systems including many oscillators very often and it is difficult to send the social lives without the phenomena.

In our previous study, we have been observing synchronization phenomena on a ladder networks and 2D lattice networks of coupled van der Pol oscillators[3]. We observed not only in-phase, anti-phase or in-and-anti-phase synchronization of steady states but also phenomena of propagating phase differences. In-and-anti-phase synchronization is a phase state which in-phase synchronization and anti-phase synchronization alternately exist. Especially, we discovered a wave which switches phase states between adjacent oscillators and continuously exists. The wave is called phaseinversion wave. We observed the phase-inversion waves which simultaneously propagate to horizontal direction and vertical direction in in-and-anti-phase synchronization on the 2D lattice shape networks by using a computer simulation. However, the phase-inversion waves which propagates to the double directions were not able to be observed by using actual circuit experiments.

In this paper, we observe phase-inversion waves which simultaneously propagate to horizontal direction and vertical direction in the in-and-anti-phase synchronization by using actual circuit experiments and numerical experiments. Propagation velocities are investigated when coupling parameters

between two adjacent oscillators are changed. A reflection mechanism of the phase-inversion waves at a corner is clarified in in-and-anti-phase synchronization for vertical direction and in-phase synchronization for horizontal direction.

\section{Circuit model}

The van der Pol oscillators are coupled by inductors $L_{0}$ as a lattice(see Fig. 1). The numbers of column and row of this system are assumed as " $N$ " respectively. We name each oscillator $\operatorname{OSC}(k, l)(0 \leq k$ and $l \leq N-1)$. An actual circuit is made as $N=5$ (see Fig. 2). A voltage of each oscillator is named $v_{(k, l)}$, and a current of a inductor in each oscillator is named $i_{(k, l)}$ (see Fig. 1). An equation of the nonlinear negative resistor is shown as Eq. (1). Circuit equations are normalized by Eq. (2). The normalized circuit equations are shown as Eqs. (3)-(7). The $\alpha$ corresponds to a coupling parameter. The $\varepsilon$ corresponds to a nonlinearity of each oscillator. This circuit is simulated by using the fourth order Runge-Kutta method and Eqs. (3)-(7).

$$
\begin{aligned}
& i_{r}\left(v_{(k, l)}\right)=-g_{1} v_{(k, l)}+g_{3} v_{(k, l)}^{3} . \\
& i_{(k, l)}=\sqrt{\frac{C g_{1}}{3 L g_{3}}} x_{(k, l)}, \quad v_{(k, l)}=\sqrt{\frac{g_{1}}{3 g_{3}}} y_{(k, l)}, \\
& t=\sqrt{L C} \tau, \quad \frac{d}{d \tau}=“ . ", \alpha=\frac{L}{L_{0}}, \quad \varepsilon=g_{1} \sqrt{\frac{L}{C}} .
\end{aligned}
$$

[Corner-top] (left: $(a, b)=(0,1)$, right: $(a, b)=(N-1, N-2)$.)

$$
\begin{aligned}
& \frac{d x_{(0, a)}}{d \tau}=y_{(0, a)}, \\
& \begin{aligned}
\frac{d y_{(0, a)}}{d \tau}=-x_{(0, a)}+\alpha\left(x_{(0, b)}+x_{(1, a)}-2 x_{(0, a)}\right) \\
+\varepsilon\left(y_{(0, a)}-\frac{1}{3} y_{(0, a)}^{3}\right) .
\end{aligned}
\end{aligned}
$$

[Corner-bottom] (left: $(a, b)=(0,1)$, right: $(a, b)=(N-1, N-2)$.)

$$
\begin{aligned}
& \frac{d x_{(N, a)}}{d \tau}=y_{(N, a)}, \\
& \frac{d y_{(N, a)}}{d \tau}=-x_{(N, a)}+\alpha\left(x_{(N-1, a)}+x_{(N, b)}-2 x_{(N, a)}\right) \\
& +\varepsilon\left(y_{(N, a)}-\frac{1}{3} y_{(N, a)}^{3}\right) . \\
& \text { [Center] }(0<k<N-1,0<l<N-1 \text {. }) \\
& \frac{d x_{(k, l)}}{d \tau}=y_{(k, l)} \text {, } \\
& \frac{d y_{(k, l)}}{d \tau}=-x_{(k, l)}+\alpha\left(x_{(k+1, l)}+x_{(k-1, l)}+x_{(k, l+1)}+x_{(k, l-1)}\right. \\
& \left.-4 x_{(k, l)}\right)+\varepsilon\left(y_{(k, l)}-\frac{1}{3} y_{(k, l)}^{3}\right) \text {. }
\end{aligned}
$$



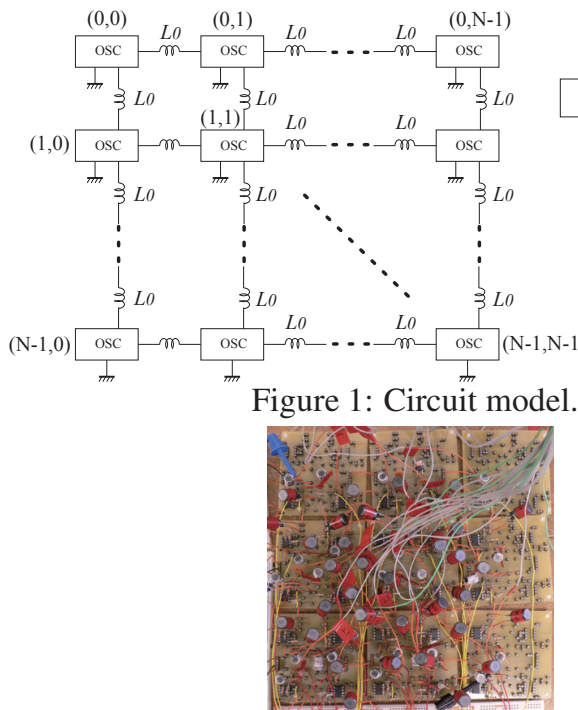

Figure 2: Photo of actual circuit.

[Edge]

(top: $(a, b)=(0,1)$, bottom: $(a, b)=(N-1, N-2)$,both: $0<l<N-1$.)

$\frac{d x_{(a, l)}}{d \tau}=y_{(a, l)}$,

$\frac{d y_{(a, l)}}{d \tau}=-x_{(a, l)}+\alpha\left(x_{(a, l-1)}+x_{(a, l+1)}+x_{(b, l)}-3 x_{(a, l)}\right)$

$$
+\varepsilon\left(y_{(a, l)}-\frac{1}{3} y_{(a, l)}^{3}\right) .
$$

(left: $(a, b)=(0,1)$, right: $(a, b)=(N-1, N-2)$, both: $0<k<N-1$.

$$
\begin{aligned}
& \frac{d x_{(k, a)}}{d \tau}=y_{(k, a)}, \\
& \begin{aligned}
\frac{d y_{(k, a)}}{d \tau}=-x_{(k, a)}+\alpha\left(x_{(k-1, a)}+x_{(k+1, a)}+\right. & \left.x_{(k, b)}-3 x_{(k, a)}\right) \\
& +\varepsilon\left(y_{(k, a)}-\frac{1}{3} y_{(k, a)}^{3}\right) .
\end{aligned}
\end{aligned}
$$

\section{Phase-invertion-waves}

A computer simulation result of existing the phaseinversion waves in in-and-anti-phase synchronization for vertical direction and in-phase synchronization for horizontal direction are shown in Fig. 3 when $N=10$. In-and-anti-phase synchronization is a synchronization phenomena which the in-phase synchronization and the anti-phase synchronization are alternately existing. The Fig. 3-A expresses an attractor of each oscillator(current vs. voltage). The Fig. 3-B expresses itinerancy of phase difference by which sum of voltages of adjacent oscillators is shown along the time(sum of voltages vs. time). Black areas are almost the in-phase synchronization. White areas are almost the anti-phase synchronization. The signs of the initial values of the voltages are shown in Fig. 4. In Fig. 3, we can observe phase-inversion waves which simultaneously propagate to horizontal direction in in-phase synchronization and to vertical direction in in-and-anti-phase synchronization.

\section{Propagation velocity}

We analyze a propagation velocity by using numerical experiments. The propagation velocity is calculated by using time of which the phase-inversion wave propagates from the 1 st row to the $N$ th row or from the 1 st column to the $N$ th column. Observation conditions are fixed as follows:

1. $N$ is fixed as 10 .

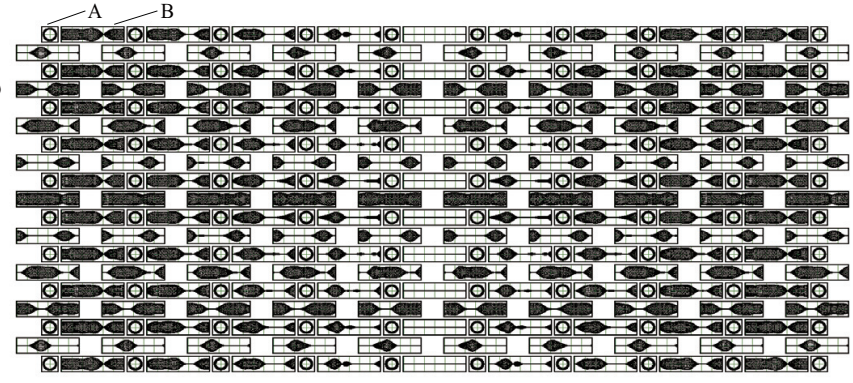

Figure 3: Computer simulation result of the phase-inversion waves in in-and-anti-phase synchronization for vertical direction and in-phase synchronization for horizontal direction $(N=10, \alpha=0.010$, and $\varepsilon=0.050)$.

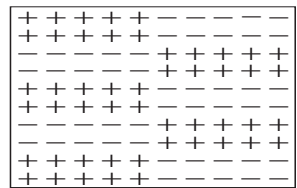

Figure 4: Sign of initial value of each oscillator of Fig. 3.
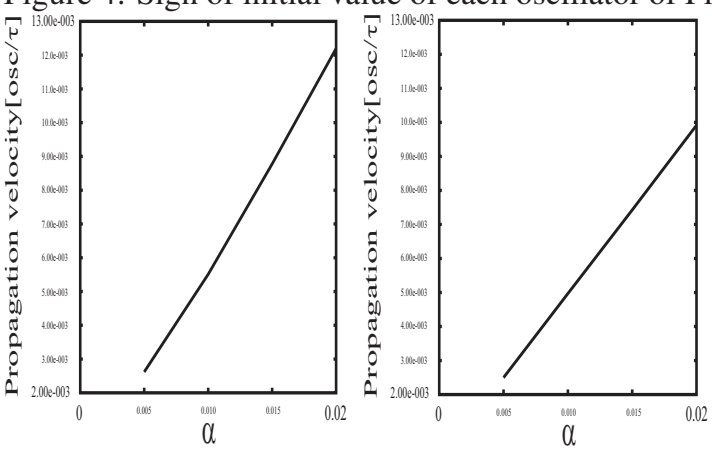

(a) Vertical phase-inversion wave. (b) Horizantal phase-inversion wave.

Figure 5: Propagation velocities of the phase-inversion wave.

2. The nonlinearity is fixed 0.080 , and the coupling parameter is changed form 0.005 to 0.020 every 0.005 .

3. Time of that the phase-inversion wave propagate from $\operatorname{OSC}(1,0)$ to $\operatorname{OSC}(1,4)$ or from $\operatorname{OSC}(0,1)$ to $\operatorname{OSC}(4,1)$ is investigated because two phase-inversion waves exist in each column or in each row.

The propagation velocity of phase-inversion wave is shown in Fig. 5. The vertical axes are the propagation velocity. The horizontal axes are the coupling parameter $\alpha$. The propagation velocity is calculated by Eq. (8).

$$
\text { propagation velocity }=\frac{M}{T} \text {, }
$$

where the number of oscillator propagating the phaseinvertion wave is assumed as " $M$," and a propagation time is expressed as " $T$." Comparison of each propagation velocity is shown in Table 1 . We can see that the propagation velocity increases with the increasing of the coupling parameter. The velocity of the phase-inversion wave in the in-phase synchronization is slower than the velocity of the phase-inversion wave in the in-and-anti-phase synchronization.

\section{Mechanisms}

We can observe a phenomenon that a vertical phaseinversion wave and a horizontal phase-inversion wave reflect at a corner. Vertical phase-inversion waves propagate in 
Table 1: Comparisons of the propagation velocities.

\begin{tabular}{|c|l|l|}
\hline$\alpha$ & $\begin{array}{l}\text { vertical in the in-and-anti- } \\
\text { phase synchronization }\end{array}$ & $\begin{array}{l}\text { horizontal in the in-and-anti- } \\
\text { phase synchronization }\end{array}$ \\
\hline 0.005 & $2.617 \times 10^{-3}$ & $2.498 \times 10^{-3}$ \\
\hline 0.010 & $5.511 \times 10^{-3}$ & $4.969 \times 10^{-3}$ \\
\hline 0.015 & $8.777 \times 10^{-3}$ & $7.424 \times 10^{-3}$ \\
\hline 0.020 & $1.220 \times 10^{-2}$ & $9.917 \times 10^{-3}$ \\
\hline
\end{tabular}

the in-and-anti-phase synchronization and horizontal phaseinversion waves propagate in the in-phase synchronization. This mechanism is analyzed by using an instantaneous frequency of each oscillator and phase differences between adjacent oscillators. The coupling parameter is fixed as $\alpha=0.01$, and nonlinearity is fixed as $\varepsilon=0.15$.

The instantaneous frequency is defined as Eq. (9).

$$
f_{(k, l)}(a)=\frac{1}{\tau_{(k, l)}(a)-\tau_{(k, l)}(a-1)},
$$

where "a" expresses the number of times of the voltage positive peak value, namely $\tau_{(k, l)}(a)$ means the time of $\operatorname{OSC}(\mathrm{k}, 1)$ as shown in Fig. 6. Similarly, $\tau_{(k+1, l)}(a)$ and $\tau_{(k, l+1)}(a)$ are defined. Some frequencies are observed in this phenomena. These frequencies are needed to consider the synchronization states for the vertical direction and horizontal direction, because this system is 2-dimensional array. Three phase states have to be considered for an oscillator in edges of 2D lattice, because the in-phase synchronization and the anti-phase synchronization are exist in same time. Therefore, three types synchronization states are observed in edges of the lattice as follows:

1. Two of three phase states are the in-phase synchronization state. Another phase state is the anti-phase synchronization state.

2. Two of three phase states are the anti-phase synchronization state. Another phase state is the in-phase synchronization state.

3. Three synchronization states are the anti-phase synchronization.

The 1 st type is called $f_{\text {iia }}$. The 2 nd type is called $f_{\text {iaa }}$. The 3rd type is called $f_{\text {aaa }}$.

The phase difference is calculated as follows. A phase difference between $\operatorname{OSC}(k, l)$ and $\operatorname{OSC}(k+1, l)$ and a phase difference between $\operatorname{OSC}(k, l)$ and $\operatorname{OSC}(k, l+1)$ are obtained. The phase differences are assumed as $\Phi_{(k, l)(k+1, l)}(a)$ and $\Phi_{(k, l)(k, l+1)}(a)$, respectively. The $\Phi_{(k, l)(k+1, l)}(a)$ and $\Phi_{(k, l)(k, l+1)}(a)$ are obtained by Eq. (10) (see Fig. 6).

$$
\begin{aligned}
& \Phi_{(k, l)(k+1, l)}(a)=\frac{\tau_{(k, l)}(a)-\tau_{(k+1, l)}(a)}{\tau_{(k, l)}(a)-\tau_{(k, l)}(a-1)} \times 2 \pi[\mathrm{rad}] \\
& \Phi_{(k, l)(k, l+1)}(a)=\frac{\tau_{(k, l)}(a)-\tau_{(k, l+1)}(a)}{\tau_{(k, l)}(a)-\tau_{(k, l)}(a-1)} \times 2 \pi[\mathrm{rad}] .
\end{aligned}
$$

Reflection mechanism at a corner is shown in Table 2(see Fig. 7). In upside of Figs. 7(a) and (b), the vertical axes are instantaneous frequency, and the horizontal axes are time. In downside of Figs. 7(a) and (b), the vertical axes are the phase difference, and the horizontal axes are time.

\section{Experimental results}

The actual circuit experimental result of $N=5$ is shown in Fig. 8. The numerical experimental result is shown in

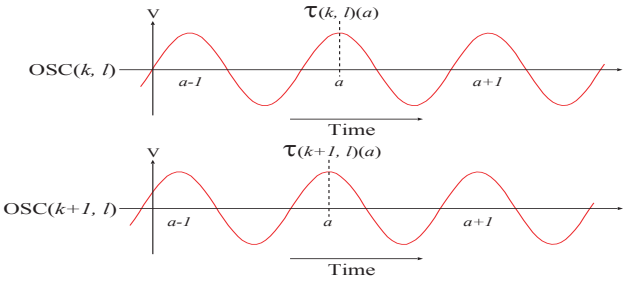

Figure 6: The detection method of the instantaneous frequencies and the phase differences.
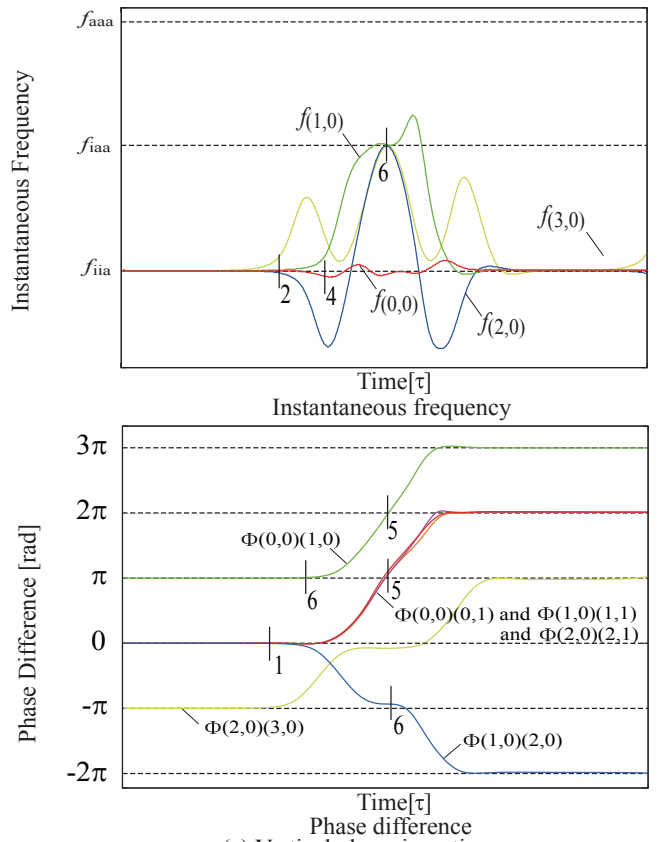

(a) Vertical phase-invertion wave.
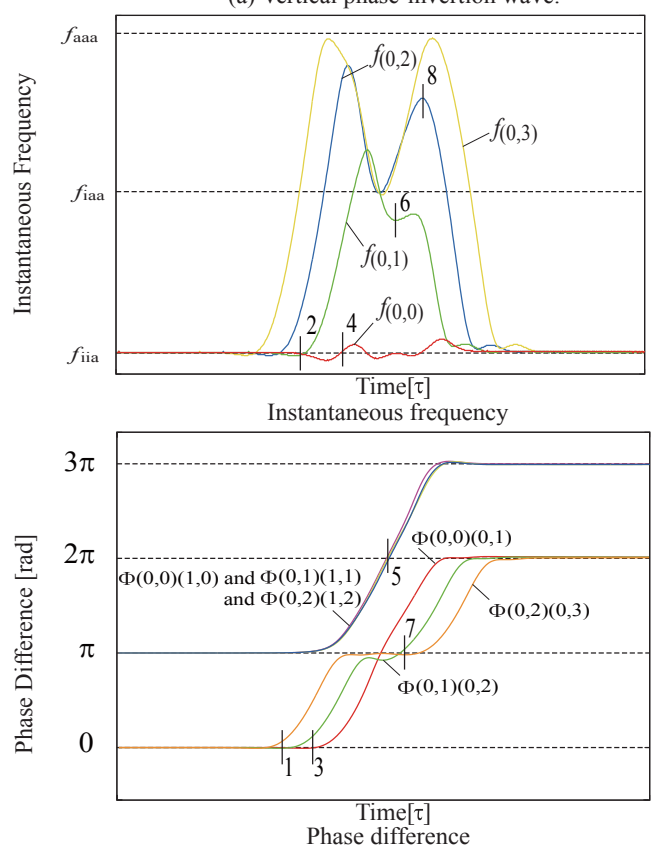

(b) Horizontal phase-invertion wave.

Figure 7: Transitions of instantaneous frequencies and phase differences by the reflection of two phase-inversion waves at a corner.

Fig. 9. We can observe phase-inversion waves which simultaneously propagate to horizontal direction and vertical direction in the in-and-anti-phase synchronization. The 
Table 2: Reflection mechanism of two phase-inversion waves at a corner (see Fig. 7).

\begin{tabular}{|c|c|c|c|}
\hline no. & Vertical phase-inversion wave & no. & Horizontal phase-inversion wave \\
\hline 0 & $\begin{array}{l}\text { At first, the instantaneous frequency } f_{(1,0)} \text { is } f_{\text {iia }} \text {, because } \\
\Phi_{(0,0)(1,0)} \text { is } \pi \text {, and } \Phi_{(1,0)(2,0)} \text { and } \Phi_{(1,0)(1,1)} \text { are } 0 \text {. The verti- } \\
\text { cal phase-inversion wave comes from } 12 \text { th row to } 2 \text { nd row in } \\
\text { the in-and-anti-phase synchronization in each column. }\end{array}$ & 0 & $\begin{array}{l}\text { At first, the instantaneous frequency } f_{(0,1)} \text { is } f_{\text {iia }} \text {, because } \\
\Phi_{(0,1)(1,1)} \text { is } \pi \text { and } \Phi_{(0,0)(0,1)} \text { and } \Phi_{(0,1)(0,2)} \text { are } 0 \text {. The horizontal } \\
\text { phase-inversion wave comes from } 11 \text { th column to } 2 \text { nd column } \\
\text { in the in-phase synchronization in each row. }\end{array}$ \\
\hline 1 & $\begin{array}{l}\Phi_{(1,0)(2,0)} \text { starts to change from } 0 \text { toward }-\pi \text { by the vertical } \\
\text { phase-inversion wave. }\end{array}$ & 1 & $\begin{array}{l}\Phi_{(0,1)(0,2)} \text { starts to change from } 0 \text { toward } \pi \text { by the horizon- } \\
\text { tal phase-inversion wave. } f_{(0,2)} \text { is changing to } f_{\text {iaa }} \text {, because } \\
\Phi_{(0,1)(0,2)} \text { is } 0, \Phi_{(0,2)(1,2)} \text { is } \pi \text { and } \Phi_{(0,2)(0,3)} \text { is changing to } \pi \text {. }\end{array}$ \\
\hline 2 & $\begin{array}{l}f_{(1,0)} \text { starts to change from } f_{\text {iia }} \text { to } f_{\text {iaa }} \text {, because } \Phi_{(1,0)(2,0)} \text { is } \\
\text { changing from } 0 \text { to }-\pi, \Phi_{(0,0)(1,0)} \text { is } \pi \text { and } \Phi_{(1,0)(1,1)} \text { is } 0 \text {. }\end{array}$ & 2 & $\begin{array}{l}f_{(0,1)} \text { starts to change from } f_{\text {iia }} \text { toward } f_{\text {iaa }} \text {, because } \Phi_{(0,1)(0,2)} \\
\text { is changing from } 0 \text { to } \pi, \Phi_{(0,0)(0,1)} \text { is } 0 \text { and } \Phi_{(0,1)(1,1)} \text { is } \pi \text {. }\end{array}$ \\
\hline 3 & $\begin{array}{l}\Phi_{(0,0)(1,0)} \text { starts to change from } \pi \text { toward } 2 \pi \text {, because } f_{(1,0)} \text { is } \\
\text { changing from } f_{\text {iia }} \text { to } f_{\text {iaa }} \text {. }\end{array}$ & 3 & $\begin{array}{l}\Phi_{(0,0)(0,1)} \text { starts to change from } 0 \text { toward } \pi \text {, because } f_{(0,1)} \text { is } \\
\text { changing from } f_{\text {iia }} \text { to } f_{\text {iaa }} \text {. }\end{array}$ \\
\hline 4 & \multicolumn{3}{|c|}{$f_{(0,0)}$ almost never changes, because $\Phi_{(0,0)(0,1)}$ is changing from 0 to $\pi$ and $\Phi_{(0,0)(1,0)}$ is changing from $\pi$ to $2 \pi$. } \\
\hline 5 & \multicolumn{3}{|c|}{$\begin{array}{l}\Phi_{(0,0)(1,0)} \text { continuously changes to } 3 \pi \text {, because the in-phase synchronization can't exist at an edge in the in-and-anti-phase synchronization } \\
\Phi_{(0,0)(0,1)} \text { continuously changes to } 2 \pi \text {, because the anti-phase synchronization can't exist at an edge in the in-phase synchronization. }\end{array}$} \\
\hline \multirow[t]{3}{*}{6} & \multirow{2}{*}{$\begin{array}{l}f_{(1,0)} \text { starts to change toward } f_{\text {aaa }} \text {, because } \Phi_{(0,0)(1,0)} \text { is chang- } \\
\text { ing to } 3 \pi \text { and } \Phi_{(1,0)(1,1)} \text { is almost } \pi \text { yet. } \Phi_{(1,0)(2,0)} \text { is start to } \\
\text { change from }-\pi \text { to }-2 \pi \text {, because } f_{(1,0)} \text { is changing toward } f_{\text {aaa }} \\
\text { and a difference between } f_{(1,0)} \text { and } f_{(2,0)} \text { is increased. } f_{(2,0)} \\
\text { starts to change toward } f_{\text {iia }} \text {, because } \Phi_{(1,0)(2,0)} \text { is changing to } \\
-2 \pi, \Phi_{(2,0)(2,1)} \text { is almost } \pi \text { yet and } \Phi_{(2,0)(3,0)} \text { is } 2 \pi \text {. }\end{array}$} & 6 & $\begin{array}{l}f_{(0,2)} \text { is higher than } f_{(0,1)} \text {, because } \Phi_{(0,1)(0,2)} \text { and } \Phi_{(0,2)(0,3)} \text { is } \pi \\
\text { and } \Phi_{(0,2)(1,2)} \text { is changing to } 3 \pi \text {. }\end{array}$ \\
\hline & & 7 & $\begin{array}{l}\Phi_{(0,1)(0,2)} \text { starts to change to } 2 \pi \text {, because } f_{(0,1)} \text { is lower than } \\
f_{(0,2)} \cdot f_{(0,1)} \text { starts to change to } f_{\text {iia }} \text {, because } \Phi_{(0,0)(0,1)} \text { is chang- } \\
\text { ing to } 2 \pi, \Phi_{(0,1)(1,1)} \text { is changing to } 3 \pi \text { and } \Phi_{(0,1)(0,2)} \text { is changing } \\
\text { to } 2 \pi \text {. }\end{array}$ \\
\hline & $\begin{array}{l}f_{(1,0)} \text { starts to change toward } f_{\text {iia }} \text {, because } \Phi_{(0,0)(1,0)} \text { changes to } \\
3 \pi, \Phi_{(1,0)(1,1)} \text { changes to } 2 \pi \text { and } \Phi_{(1,0)(2,0)} \text { is changing to }-2 \pi\end{array}$ & 8 & $\begin{array}{l}f_{(0,2)} \text { can't arrive at } f_{\text {aaa }} \text { and starts to change to } f_{\text {iaa }} \text {, because } \\
\Phi_{(0,1)(0,2)} \text { is changing to } 2 \pi \text {. Because } f_{(0,2)} \text { is changing to } f_{\text {iaa }} \text {, } \\
f_{(0,2)} \text { is lower than } f_{(0,3)} \text { and } \Phi_{(0,2)(0,3)} \text { starts to change to } 2 \pi \text {. } \\
f_{(0,2)} \text { continuously changes to } f_{\text {iia }} \text {, because } \Phi_{(0,1)(0,2)} \text { is chang- } \\
\text { ing to } 2 \pi \text {. } \Phi_{(0,2)(1,2)} \text { is } 3 \pi \text { and } \Phi_{(0,2)(0,3)} \text { is changing to } 2 \pi \text {. }\end{array}$ \\
\hline
\end{tabular}

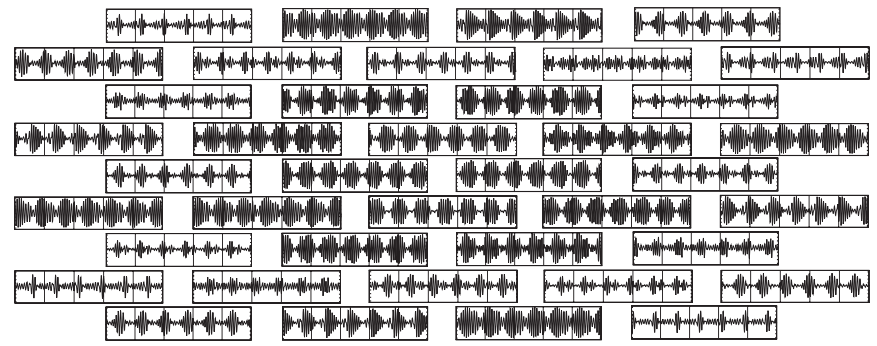

Figure 8: Circuit experimental $\operatorname{result}\left(N=5, R_{1} \simeq R_{2} \simeq 5.1\right.$ $\mathrm{k} \Omega, R_{3} \simeq 1.6 \mathrm{k} \Omega, C \simeq 5.6 \mathrm{nF}, L \simeq 10 \mathrm{mH}$ and $\left.L_{0} \simeq 20 \mathrm{mH}\right)$.

propagation velocity of actual circuit is normalized for time (propagationvelocity $\times \sqrt{L_{0} C}$ ) because the velocity has to be compared with simulation result. When $L_{0}$ is $30 \mathrm{mH}, \alpha$ is around 0.33 . When $L_{0}$ equals $20 \mathrm{mH}, \alpha$ is 0.50 . The normalized propagation velocity of $L_{0}=30 \mathrm{mH}$ is 0.92 times of the normalized propagation velocity of $L_{0}=20 \mathrm{mH}$. In simulation results, the propagation velocity of $\alpha=0.5$ is 0.90 times of the propagation velocity of $\alpha=0.33$. We can observed almost same values between simulation results and experimental results. We can think that our simulation results can be observed in actual experiments.

\section{Conclusion}

We clarified that phase-inversion waves simultaneously propagate to horizontal direction and vertical direction in inand-anti-phase synchronization by using actual circuit experiments and numerical experiments. To increase of propaga-

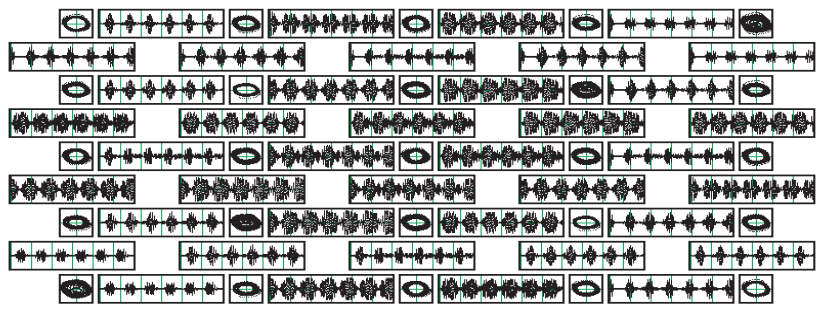

Figure 9: Computer simulation $\operatorname{result}(N=5, \alpha=0.50$ and $\varepsilon=0.80)$.

tion velocity was made clear when the coupling parameters are increased. Furthermore, the reflection mechanism of the phase-inversion waves was analyzed at a corner.

Acknowledgements

This work was supported by JSPS KAKENHI Grant Number 25730152 .

\section{References}

[1] C. M. Gray, "Synchronous Oscillations in Neuronal Systems: Mechanisms and Functions," Journal of Computational Neuroscience 1, pp. 11-38, 1994.

[2] How-Foo Chen; Jia-Ming Liu; "Unidirectionally Coupled Synchronization of Optically Injected Semiconductor Lasers," IEEE Journal of Selected Topics in Quantum Electronics, vol. 10, issue 5, pp.918-926, 2004.

[3] S. YANANE, M. YANAUCHI, "Propagation and Reflection of Phase Differences on a Lattice of Coupled Oscillators," Proc. ISCAS'09, pp.1895-1898, May 2009. 